\title{
RESEARCH ON NATIONAL 1:50000 TOPOGRAPHIC CARTOGRAPHY DATA ORGANIZATION
}

\author{
Chenchen $\mathrm{Wu}^{\mathrm{a}, *}$, Jianwei Liu ${ }^{\mathrm{a}}$, Jianjun $\mathrm{Liu}^{\mathrm{a}}, \mathrm{Zhao} \mathrm{Li}^{\text {a }}$ \\ ${ }^{a}$ National Geomatics Center of China, 28 Lianhuachixi Road, Beijing, Chian - (wucc, liujw, liujj, lzh)@nsdi.gov.cn
}

\author{
Commission IV, WG IV/2
}

KEY WORDS: Database-driven Cartography, Data Organization, Topographic Map, Terrain data

\begin{abstract}
:
Database-driven cartography technology is just appeared in China. In China, it's the first time to use this technology in such a large national terrain information database. Especially in the particular 1:50000 scale, it will face a more complex situation. To effectively address the problem, with the database-driven cartography mechanism, it is very necessary to design scientific and rational model for data organization and presentation mechanisms, to ensure that the rich geographic information of terrain data can be expressed correctly, completely and beautifully in the topographic map. This paper mainly introduces the general design of data organization model of the cartography data. It also designed and developed national 1:50000 topographic cartography production system, and gave out related application example as a test of the idea mentioned. The application example proves that the data organization model designed here is feasible and efficient in nation 1:50000 topographic map production, and greatly reducing the workload of manual editing.
\end{abstract}

\section{INTRODUCTION}

China is developing rapidly, with the changing feature, especially residential areas, roads and so on. Therefore, update of topographic information database and paper topographic map is very urgent. Currently, national 1:50000 topographic information database update project in China's eleventh five-year plan is nearly complete. The result data is aggregated and built database gradually. On this basis, the paper topographic map production has gradually carried out. To take full advantage of the updated results of the terrain information database and enhance the production efficiency of topographic maps, national 1:50000 topographic cartography productions using a database-driven cartography technology.

Database-driven cartography technology is just appeared in China. It mainly referring to the integration process that terrain database directly converted to the cartography database. The entire process is driven by terrain information database, and derives cartographic information according to the data model.

This process contains changes and contradictions. Terrain data is mainly a description of the space topology and properties storage, expressed in the form of point, line and polygon, and stored in the database. But cartography data expresses a wealth of information of earth surface, much more than terrain data. All the spatial information and attribute information included in terrain data are translated into the language of the map, to describe the surface of land. Therefore, in the transformation from terrain data to cartography data, there are many processes need to undergo such as expansion, reorganization, extraction, mining and so on, to solve the differences and contradictions between terrain data character and cartography data requirements.

In China, many thematic maps or small databases are using such a cartography technology. But it's the first time to use this technology in such a large national terrain information database.
Especially in the particular 1:50000 scale, it will face a more complex situation. 1:50000 is the most complicate one among the national 8 base scale. It contains most abundant expression of information, and most diverse content. Therefore, the national 1:50000 terrain data are also the most complex, multi-layer, multi-element, attribute rich.

To effectively address the problem, with the database-driven cartography mechanism, it is very necessary to design scientific and rational model for data organization and presentation mechanisms, to ensure that the rich geographic information of terrain data can be expressed correctly, completely and beautifully in the topographic map.

This paper does corresponding research of 1:50000 topographic map expression system, detailing the database-driven national 1:50000 topographic cartography expression mechanisms. The study involves the entire process that terrain data transfer to the paper topographic map, including issues as data organization, feature relationship, symbol design. And the final result data of national 1:50000 terrain information database update were taken as the experimental area to carry out relevant tests.

\section{RESEARCH AND ANALYSIS}

The national database-driven production of 1:50000 topographic maps, is based on the update of national 1:50000 terrain information database completed recently. Therefore, the production of 1:50000 topographic map takes achievement of the update work as source database. The source database is consist of a total of nine data sets, thirty four layers in different data type, and more than three hundred kinds of features. Achievement data of the terrain information database updated is stored in Oracle by using ArcSDE.

Under database-driven cartography mechanism, cartography data is the expansion of terrain data. It needs to be organized as well as possible, expressed as reasonable as 
possible, base on data structure of source terrain data. So the cartography database can meet china's standard of topographic map, and traditional habit to read and understand map.

As shown in Fig.1, data from the origin database is expressed by simple points, lines and polygons. After a series of graphics expansion, Fig. 2 forms a map which gives a lot of information.

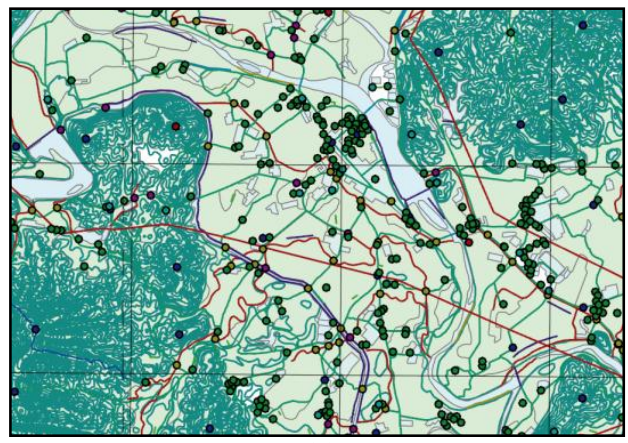

(a)

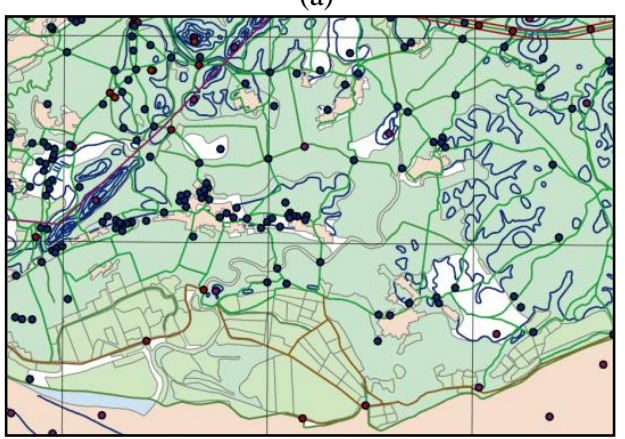

(b)

Fig.1 Source terrain data

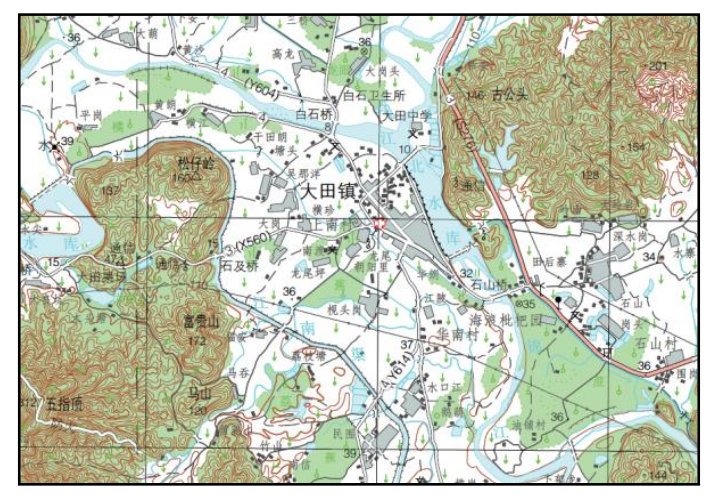

(a)

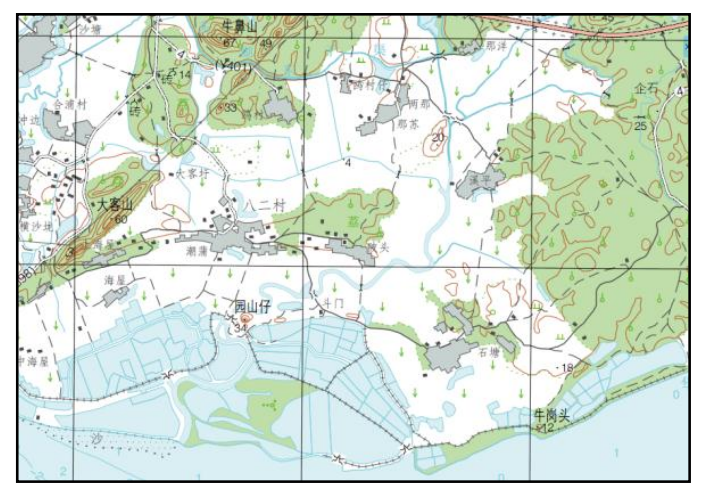

(b)

Fig.2 Topographic map data
Research work of cartography data organization has carried out through observation of technical methods of production used home and abroad. It analyzed data structure of national 1:50000 terrain information database in detail, and carefully studied china's 1:50,000 topographic map specification and relevant standards. With the actual production experience from production unit, comparative analysis and system summary of difference between terrain data status and topographic map data requirements were carried out. Detailed analysis of the national production of 1:50000 topographic cartography data in the organization and expression mechanism has been carried out too. The main data organization structure of cartography data is shown below.

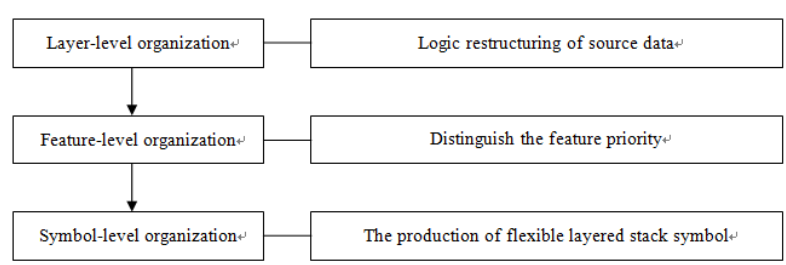

Fig.3 Organization hierarchy structure of cartography data

\subsection{The layer-level organization}

About layer, there are many types of definitions. Professional graphics software such as CorelDraw most means concept that layers is consist by graphics with the same color, style, or performance effects. But layer discussed in this article is mainly divided according to feature type and geometric characteristics. And it could also stores feature properties. According to feature type, it can be divided into 9 categories. There are control point, water, road, boundary, residential areas, terrain, vegetation, pipeline, and place names. According to geometric characteristics, it can be divided into 3 classes. There are point, line and polygon. Combining two aspects, the terrain data is divided into 34 layers, cartography data generally retained these 34 layers.

During the database-driven cartography, the hierarchical structure and overall framework of cartography data are high degree depend on the source database. After a detailed analysis of the national 1:50,000 topographic database update results, 34 feature layers were split, sorted, and grouped. At the same time, certain relationships can exist between layers. It allows interruption, cover, topological manipulation between layers, without changing the real data. So it will be easier to make the effect of drawing specifications meet the standard of topographic maps.

(1) 4 layer group

Terrain layer has 34 layers, and it will be more complicated after split to the cartography data layer. So it is very necessary to divide layers to different group. According to feature's geometric character, it can be divided into annotations and symbols, while the symbols can be divided into point, line and polygon. From top to bottom, 34 layers can be divided into 4 groups. They are annotation, point, line, and polygon. These groups are physically stored as feature class group. 


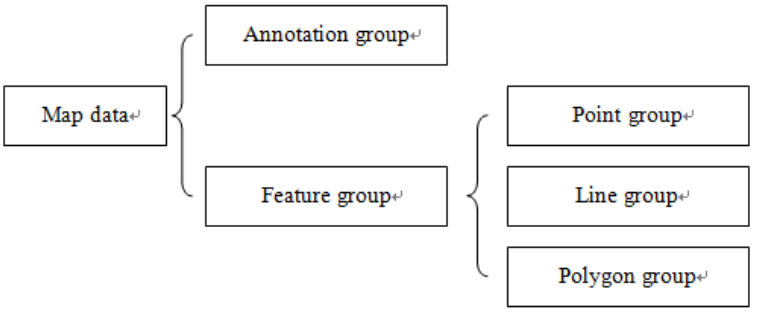

Fig.4 layer group of cartography data

(2) Organization in each layer group

From 34 layers of source database to the cartography layer, there were split, sort, and group three steps. This process is cartography data transfer from physical storage to logical organization. The following figure shows, which layer each layer group contains, on the other hand, the underpass and overpass relationships between layers within the group.

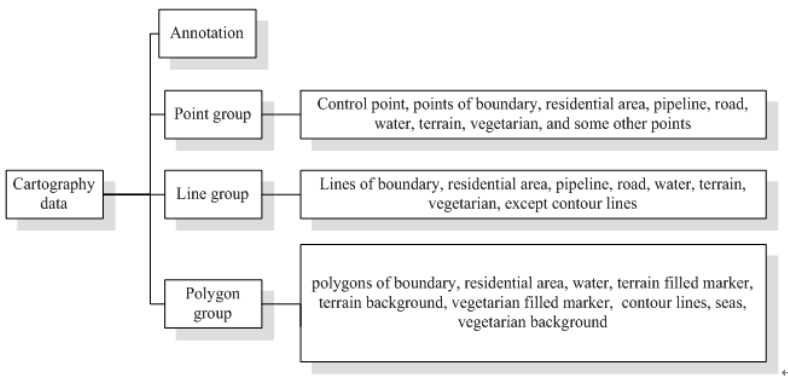

Fig.5 layer organization in each group

The four group of layers discussed above each contains different number of layers, which physical stored as several feature class. The underpass and overpass relationships are reflected by logical organization of these topographic map layers.

Annotation group and point group: Among them, each layer in annotation and point group has the same priority. Layers in this two group can neither overpass nor underpass. So there is no fixed overpass or underpass logic relationship in annotation group and point group.

Line group: priority of layer is important and obvious in this group. According to feature characteristics of the source database, we design a logical organization of the line group shown in the figure above. Line group, in accordance with the feature class of the relationship of overpass and underpass between features, can arrange follow the order from top to bottom: Lines of boundary, residential area, pipeline, road, water, terrain, vegetarian, except contour lines. Beside, the linear feature of water is put into the polygon group, due to its special characteristics.

Polygon group, from top to bottom as follows: boundary, residential area, water, filled marker of terrain, background of terrain, filled marker of vegetation, contour lines, seas, background of vegetation. Although the geometric form of contour lines is linear, but it reflects meaningful information for the surface shape. And it has low priority, so that all other features can overpass it. So the contour lines were put at the entire data structure on the end.

(3) The relationship between layers

Between the layers talked about above, there are not only logical link, but also the associated physical and cartographic relationships. It is mainly reflected in two aspects: derivation and mask.
Annotation layer and physical feature layer where annotation derived from has certain relationship.

And if a layer is masked by another layer, it is drawn underpass the other layer. This is the mechanism of mask between. When a mask is added to one layer, it looks like this layer is cut off by some other layers.

\subsection{The feature-level organization}

This chapter describes the organization of features in each layer. The feature-level organization is mainly about two relationships between features, one is feature priority and the other one is symbol integration.

Feature priority means the level of drawing order of feature symbology. Features in higher level are drawn upon the lower ones. For example, feature level of high way is higher than normal road, so high way is drawn above any other roads on the topographic map. No roads can cross high road except there is a bridge or a culvert. The feature priority represents an overpass or underpass relationship between features in the same layer.

The other hand, symbol integration lets you create special cartographic effects. This lets you create, for example, a reference map with cased-line streets, and where these streets intersect, you can either blend their symbol, representing connectivity, or not, representing an overpass or underpass. In a real-world example, if your highway symbol is integrated, this means that all your highway features will be connected when they connect to each other on the map. you can specify that your highway features only show the connectivity effect when they intersect your highway ramp features (all symbolized with your highway ramp symbol). However, when highway features intersect all other street types, for example, surface streets, they will not be connected.

Through two relationships discussed above, features in the same layer can be organized to a reasonable order. A feature-level organization data model can be designed according to the topographic map tradition and the real data characters, to decide every feature's drawn order and whether to be integrated.

\subsection{The symbol-level organization}

Because it is the national standard topographic maps, all symbols are strictly conform to national standards, do not need additional design or artistic edit. So this paper only considers the symbol's implementation mechanism, and an efficient way to achieve national standards for each of the symbols.

For many graph edit software, symbol design is an important foundation, but most of them take each symbol as a whole to design. Once encounter complex symbols or change of symbols, the whole design work and edit process need to roll over again. But symbol-level organization discussed here allows hierarchical symbol, which means one symbol can divide to several symbol layers. Each layer can be drawn with one factor in graphics libraries are referenced in the software. And the factor is taken as one part of the whole symbol. Then different geometry effect can be set to these symbol layers to design a combination symbol which looks like symbols in national standard, similar to the jigsaw puzzle.

Though symbol layers' different geometry effect, the realization of the symbol will be more flexible and convenient and may have more possibilities. In case of a large number of updates and symbol changes, you can simply modify the 
symbol factors in a layer, without having to re-create the whole symbol.

\section{EXPERIMENT AND APPLICATION}

The final experiment application system is realized according to the system general design plan described above. It adopted ArcGIS9.3.1 as the secondary development platform. According to data model of cartography data operation, the experiment was carried on layer-level, feature-level and symbol-level.

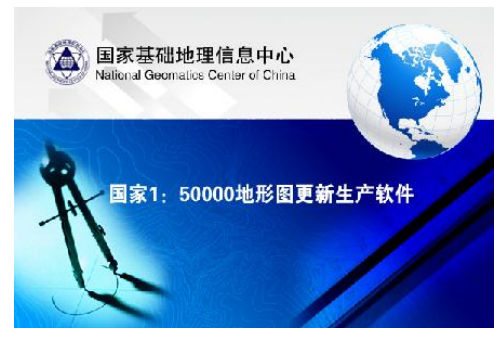

Fig.6 Experiment system boot screen

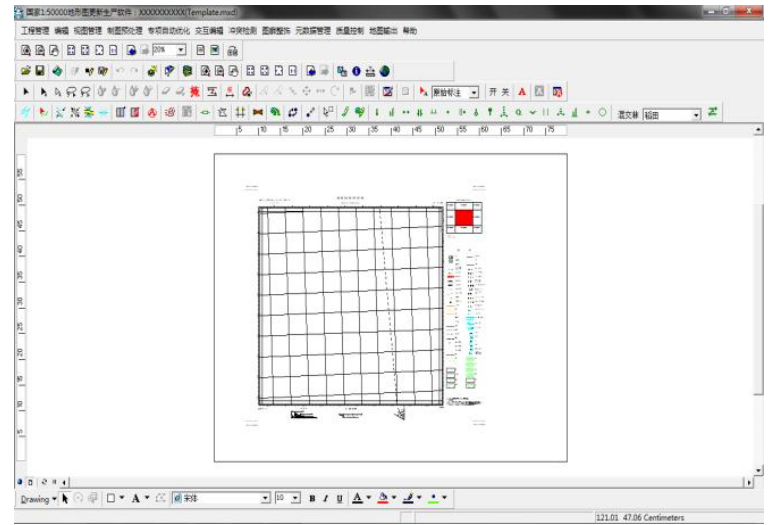

Fig.7 Initial user interface of a cartography project

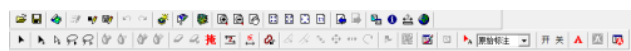

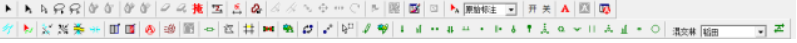

Fig.8 Toolbars of the experiment system

\subsection{Experiment on layer-level}

As discussed in 2.1, the layer-level organization is a logic restructure of source data. In experiment system, it is stored in project document. When a cartography project is open, the document will auto add data to relative layers. It is carried through the feature's "GB" code (an attribute item of feature) and its physical layer name.

Fig.9 and Fig.10 shows the layer organization original terrain database and the layer organization of cartography data. In Fig.9(a), contour lines were drawn cross roads and residential areas. And in Fig.10(a), a river was drawn overpass a road. After reorganized the layers according to the layer-level organization data model, these graphic conflicts have been solved, as shown in Fig.9 (b) and Fig.10(b).

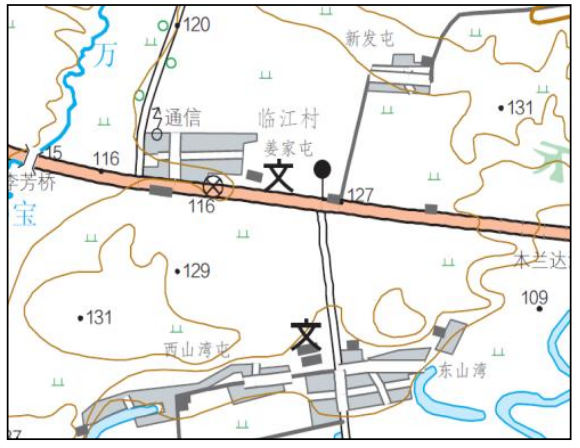

(a)

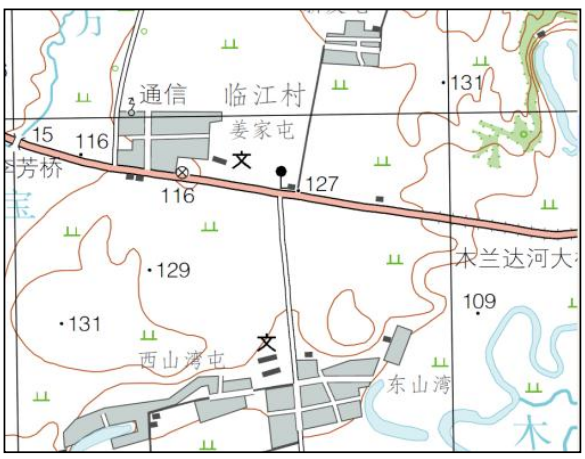

(b)

Fig.9 Conflicts between contour line and residential area solved by layer-level organization

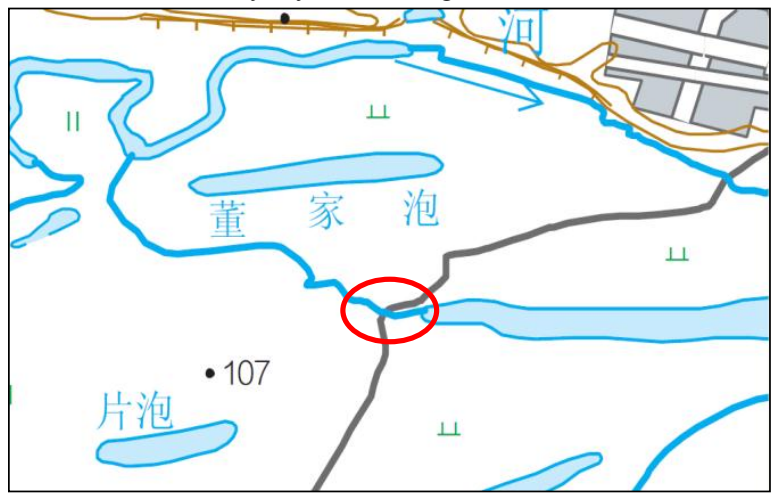

(a)

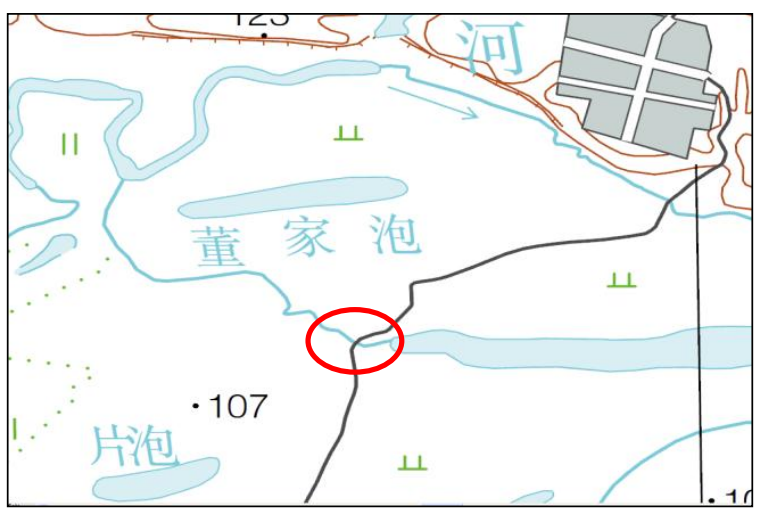

(b)

Fig.10 Conflicts between river and road solved by layer-level organization 


\subsection{Experiment on feature-level}

According the feature-level organization discussed before, a data model can be designed, like an expert system which stores the features' relationship. This data model is stored in the layer's attribute. As Fig. 11 shows, it is a list with feature code, symbol and some numbers on it. The "Label" means the feature's name, while the last three columns denote different kinds of feature priority and symbol integration.

\begin{tabular}{|c|c|c|c|c|c|c|c|}
\hline Symbol & & Layer Name & Label & -1. & .2 . & .3 . & $\hat{\wedge}$ \\
\hline$=$ & $\mathbf{v}$ & HYDA_1 & 210200 & 2 & 19 & & \\
\hline$[\cdots$ & $\mathbf{v}$ & HYDA_1 & 210301 & 2 & 17 & 18 & \\
\hline $\begin{array}{lll}* & \cdots & \cdots \\
\end{array}$ & v & HYDA_1 & 210302 & 2 & 16 & & \\
\hline & v & HYDA_1 & 220100 & 2 & 15 & & \\
\hline & - & HYDA_1 & 220200 & 2 & 14 & & \\
\hline$\because-\cdots$ & . & HYDA_1 & 230300 & 2 & 10 & 11 & \\
\hline & - & HYDA_1 & 240101 & 2 & 9 & & \\
\hline & . & HYDA_1 & 240102 & 2 & 8 & & \\
\hline & V & HYDA_1 & 261000 & 2 & 7 & & \\
\hline & - & HYDA_1 & 230101 & 2 & 3 & & \\
\hline & $\mathbf{v}$ & HYDA_1 & 230200 & 2 & 3 & & \\
\hline & & HYDA_1 & 210104 & 2 & & & \\
\hline & & HYDA_1 & 260200 & 2 & & & \\
\hline & . & HYDA_1 & 230102 & 0 & 2 & & \\
\hline & - & HYDA_1 & 250700 & 0 & 2 & & \\
\hline & iv & HYDA_1 & 221000 & 0 & 0 & & \\
\hline$y=-1$ & & LVחA 1 & 24. & $n$ & n & & $\underline{v}$ \\
\hline \multicolumn{3}{|c|}{ S witch to Default View } & & \multicolumn{4}{|c|}{ Abbout Symbol Levels } \\
\hline & & & & & OK & & icel \\
\hline
\end{tabular}

(a) water area layer

\begin{tabular}{|c|c|c|c|c|c|c|}
\hline Symbol & Layer Name & Label & -1. & .2 . & .3. & 슨 \\
\hline - & LRDL & gaosu & 5 & 23 & 23 & \\
\hline$-\mathrm{V}$ & LRDL & 420101 & 5 & 23 & & \\
\hline$-\div$ & LRDL & 420102 & 5 & 22 & & \\
\hline$\longrightarrow$ & LRDL & 420201 & 5 & 21 & & \\
\hline$-\div$ & LRDL & 420202 & 5 & 20 & & \\
\hline v & LRDL & 420301 & 5 & 19 & & \\
\hline $\mathbf{v}$ & LRDL & 420302 & 5 & 18 & & \\
\hline$\checkmark$ & LRDL & 420400 & 5 & 17 & & \\
\hline$\longrightarrow$ & LRDL & 420500 & 5 & 16 & & \\
\hline$--v$ & LRDL & 430200 & 5 & 14 & 15 & \\
\hline$\longrightarrow$ & LRDL & 430501 & 5 & 13 & & \\
\hline$-\mathbf{v}$ & LRDL & 430502 & 5 & 12 & & \\
\hline v & LRDL & 430503 & 5 & 11 & & \\
\hline $\mathbf{v}$ & LRDL & 420800 & 5 & 10 & & \\
\hline$\checkmark$ & LRDL & 430600 & 5 & 9 & & \\
\hline----- & LRDL & 440200 & 5 & & & \\
\hline -..... & $10 \mathrm{OnI}$ & Aงกวกก & a & & & $\underline{v}$ \\
\hline \multicolumn{2}{|c|}{ Switch to Default View } & & \multicolumn{4}{|c|}{ About Symbol Levels } \\
\hline & & & & $\mathrm{OK}$ & & \\
\hline
\end{tabular}

(b) road layer

Fig.11 feature-level organization data model of layers

Experiment of feature-level of water, is mainly in the person of symbol integration. The data model set symbol integration to polygon features in water area layer, except lakes and ponds. As shown in Fig.12, rivers consist of different polygons merge at intersect. After the integration the edge shared by neighbor polygons disappeared.

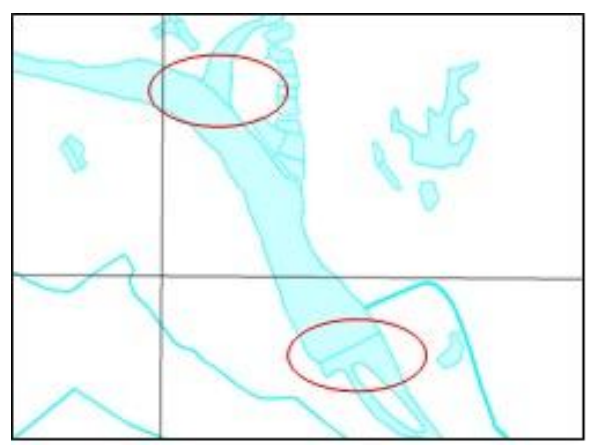

(a)Before integration

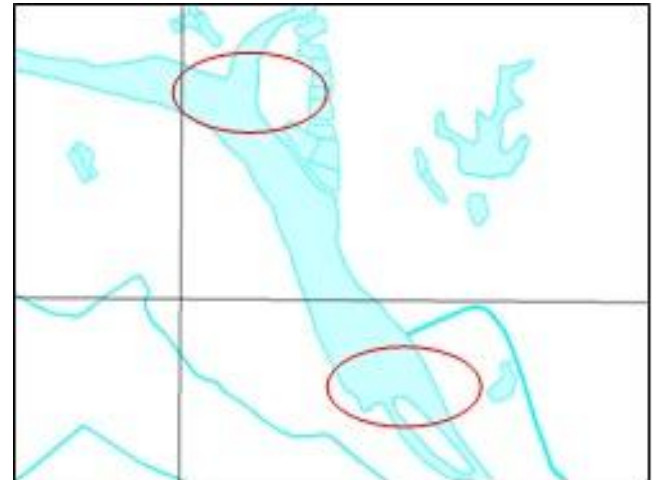

(b) After integration

Fig.12 Feature-level organization in water area layer

The experiment of feature-level organization is also carried out in road layer. It refers to both feature priority and symbol integration. The feature priority of road layer is listed in the table above, shows different grades of roads. The symbol integration set to roads keep the connectivity of roads. Fig.13 (a) and (b) are the contrast before and symbol integration. And (c) and (d) add set of feature priority to the contrast of symbol integration. The yellow road has higher priority than other roads, so it was drawn overpass others. And all intersects of roads have been merged by symbol integration to realize the connectivity of roads.

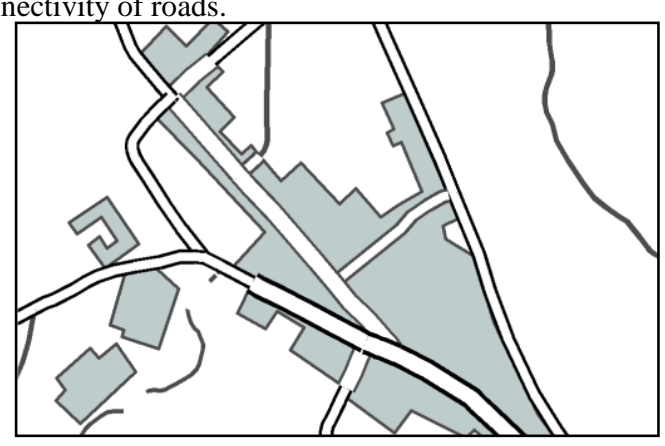

(a)

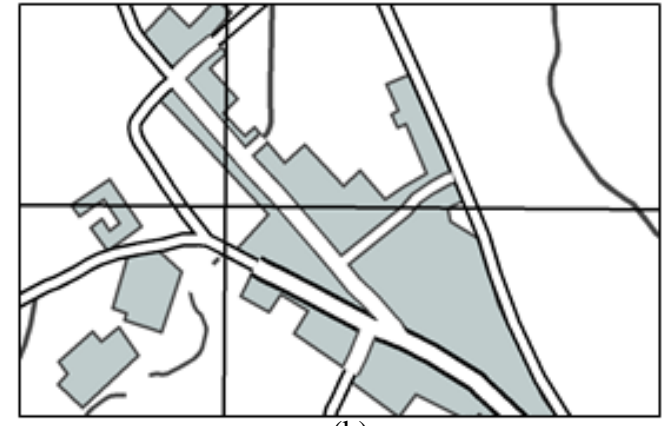

(b)

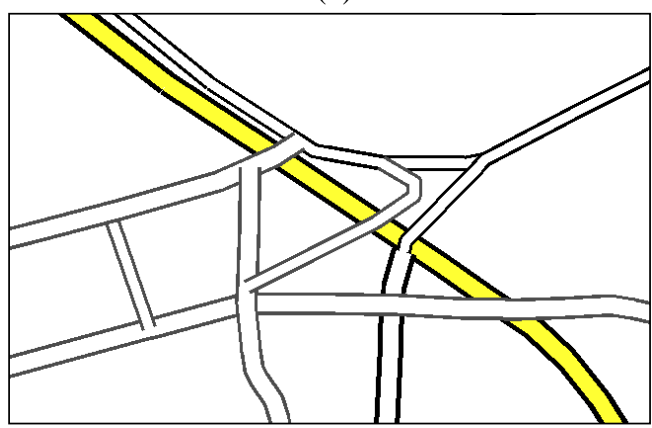

(c) 


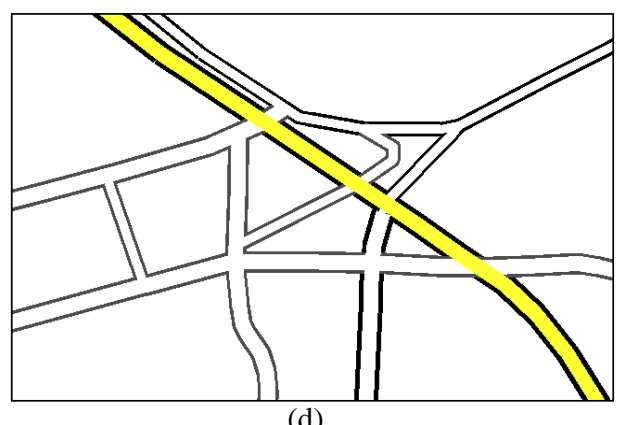

(d)

Fig.13 Feature-level organization in road layer

\subsection{Experiment on symbol-level}

Take wire net and dune as examples, the symbol-level organization is very flexible and efficiency.

As shown in Fig.14(a), this is symbol of wire net. In most graphic software, to realize this symbol, it needs to copy the "+" many times. In the experiment system, the graph factor "+" is only edit one time and set a step length in one symbol layer. It will auto drawn many times in certain pattern along the line, as shown in Fig.14(b). Along with the other symbol layer, the wire net can be realized.

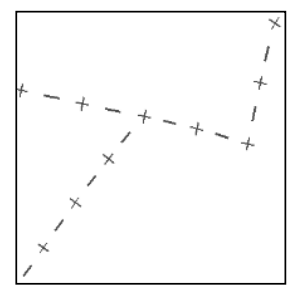

Fig.14 (a) Symbol of wire net

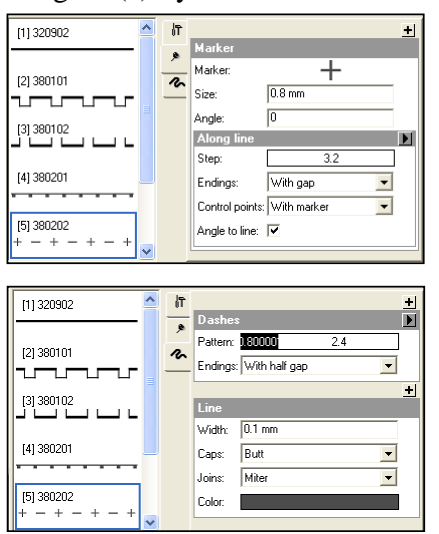

Fig.14(b) Two symbol layer of wire net

For polygon symbol, symbol layer is even more important. As shown in Fig.15, filled marker of dune is automatic repeat. Through individual parameters (Xstep, Ystep) settings shown in Fig.16, you can achieve different surface effects. As Fig.15 (b) shows, the dune's filled marker has repeat more frequently after reducing the Xstep and Ystep, instead of design another whole symbol for dune.

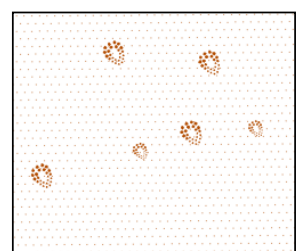

(a)

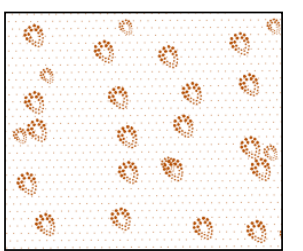

(b)
Fig.15 Different effect of dune symbol

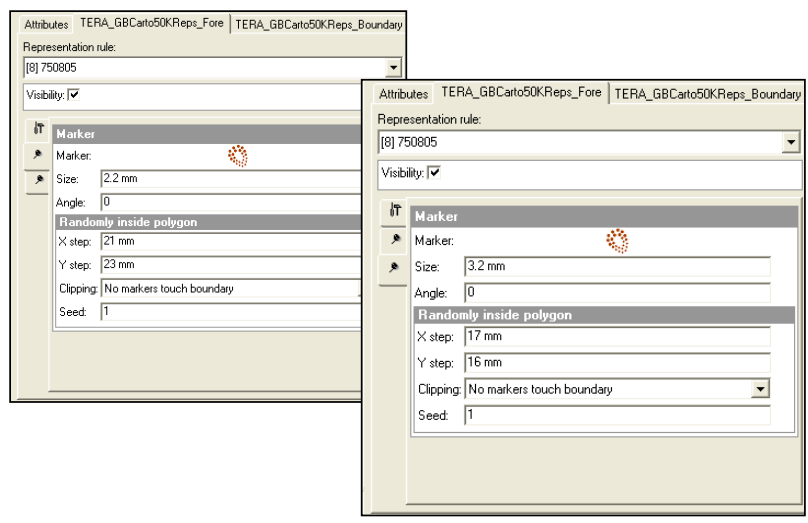

Fig.16 Two symbol layers of dune

\section{CONCLUSION}

This paper mainly introduces the general design of data organization model of the cartography data, database-driven cartography technology. It also designed and developed national 1:50000 topographic cartography system, and gave out related application example as a test of the idea mentioned. The application example proves that the data organization model designed here is feasible and efficient in nation 1:50000 topographic map production, and greatly reducing the workload of manual editing.

Database-driven cartographic data and GIS technology is a good combination of terrain data and cartography data. It includes data organization model for the perfect expression of cartographic information. Prototype of the experimental system has been applied to Chinese updated version 1:50000 topographic map production in several provinces. In the future, different data organization model can be designed according to different database structure and requirements of different scale, to carry forward multi-scale database-driven cartography production.

\section{References}

Compilation specifications for national fundamental scale maps-Pat 3:Compilation specifications for1:50000 1:25000 topographic maps, China, 2009

Mario A. Gomarasca, Elements of Cartography, 2009, Basics of Geomatics, Pages 19-77

Marco Roggero, Basics of Geomatics , 2009, 19-77, DOI: 10.1007/978-1-4020-9014-1_2 Applied Geomatics, 2009, Volume 1, Number 4, Pages 131-140

Computer Cartography, 2008, Encyclopedia of GIS, Part 6, Page 112

Computer Cartography, 2008, Encyclopedia of GIS, Part 6, Page 112

Michael P. Peterson, Elements of Multimedia Cartography, 2007, Multimedia Cartography, Pages 63-73

Andreas Neumann, Web Mapping and Web Cartography, 2008, Encyclopedia of GIS, Part 26, Pages 1261-1269 
Alberto Giordano and Lance Wisniewski , Teaching Cartography on the Web with a Multimedia GIS: A New Solution, Lecture Notes in Geoinformation and Cartography, 2008, International Perspectives on Maps and the Internet, Part C, Pages 219-238

ESRI online help 